\title{
Quantifying zooplankton species: use of richness estimators
}

\author{
Maria Carolina de A. Castilho', Maria José dos S. Wisniewski², Célio Wisniewski \& Érika dos S. Silva²
}

\author{
1. Departamento de Zoologia, Instituto de Biociências, Universidade Estadual Paulista, Caixa Postal 510, 18618-000, Botucatu, SP, Brazil. \\ (mariacarolcastilho@gmail.com) \\ 2. Instituto de Ciências da Natureza, Universidade Federal de Alfenas, 37130-000, Alfenas, MG, Brazil. \\ 3. Instituto de Ciências Exatas, Universidade Federal de Alfenas, 37500-000, Alfenas, MG, Brazil.
}

\begin{abstract}
Richness estimators (Jackknife 1, Bootstrap, Chao 1 and ACE) were used to relate zooplankton species richness with amount of water collected per sample and number of samples throughout the year for the limnetic region of Sapucai River compartment of Furnas reservoir, state of Minas Gerais, Brazil. Seven $100 \mathrm{~L}$ samples were collected in sequence using a motor pump, and seven $70 \mathrm{~L}$ samples were collected in sequence using a plankton net ( $68 \mu \mathrm{m}$ mesh size) in vertical hauls, to totalize $450 \mathrm{~L}$, in three stations of the reservoir. Twelve monthly samplings were carried out over a year. The assessment of richness was made by analyzing the asymptotic behavior of the estimator curves. The samplings reached the asymptote from $350 \mathrm{~L}$ of collection with trawls and $400 \mathrm{~L}$ using a suction motor pump and reached the plateau on the 8th collection, which included both dry and rainy seasons. Regardless of the type of sampling, the volume of $400 \mathrm{~L}$ and eight sessions throughout the year is enough to register $90 \%$ of the zooplankton richness in the environment.
\end{abstract}

KEYWORDS. Biodiversity, Cladocera, Rotifera, sampling effort, aquatic environments.

RESUMO. Quantificando espécies zooplanctônicas: uso de estimadores de riqueza. Buscando relacionar a riqueza de espécies zooplanctônicas com o volume de água coletado por amostra e com o número de amostragens ao longo do ano para a região limnética do compartimento do Rio Sapucaí no reservatório da UHE de Furnas, Estado de Minas Gerais, Brasil, foram utilizados estimadores de riqueza (Jackknife 1, Bootstrap, Chao 1 e ACE). Sete amostras de 100 litros foram coletadas com moto-bomba de sucção, e sete de 70 litros utilizando rede de plâncton de 68 m e arrastos verticais em um ponto do reservatório. Foram realizadas doze coletas mensais ao longo de um ano, nas quais foram coletados 450 litros de água por amostra, em três pontos do reservatório de Furnas. A avaliação da riqueza foi feita analisando o comportamento assintótico das curvas dos estimadores. Estes atingem a assíntota a partir de 350 litros para a coleta feita mediante arrastos e com 400 litros com utilização de moto-bomba de sucção, alcançando o platô na 8 coleta, tendo sido abrangidas as estações seca e chuvosa. Independente do tipo de amostragem, o volume de 400 litros e oito coletas ao longo do ano mostra-se suficientes para registrar $90 \%$ da riqueza zooplanctônica do ambiente.

PALAVRAS-CHAVE. Biodiversidade, Cladocera, Rotifera, esforço amostral, ambientes aquáticos.

Biological diversity can be understood at three levels: species, habitat and genetic diversity. Diversity indices use the density and richness of species and infer on the distribution equitability of species in the environment (BEGON et al., 2007; Melo, 2008).

Knowledge of aquatic diversity is fundamental to implement conservation programs and rational use of resources, to assess environmental impact for licensing of hydroelectric power plants and to regulate fish farm areas (King \& Porter, 2005; Eskinazi-SAnT'Anna et al., 2005; SAntos, 2006; Pinto-Coelho, 2004; Rocha et al., 2010; SANTOS-WisniEWsKi et al., 2011; BRITo et al., 2011). These inventories become important because the degradation of natural ecosystems and biodiversity loss have increased in recent years.
To identify and count every species of a study area is very complex. Ecological studies search to estimate the species richness of these environments by sub-samples and the use of statistical techniques (MuIRHEAD et al., 2006; Melo, 2008). Hence, the standardization of procedures and sampling effort is indispensable to reduce uncertainties and to make possible the comparison of species richness between different study environments (GOTELLI \& CowELL, 2001). Among statistical techniques, non-parametric richness estimators, such as Chao, ACE and Jackknife estimators, have been used to improve sampling. These are based on the occurrence of rare species that appear in few samples or in low density (MAGURRAN, 2011). Other estimators, such as Bootstrap, give the same value for every species collected, whether they are rare or common, to estimate the 
total richness (SANTOS, 2006).

The methodology and equipment used in samplings can interfere in species richness measurements, for example, the mesh size of the net used to collect aquatic organisms should consider organism size and escape capacity. In the same way, the collection procedure, such as the use of suction pumps or vertical hauls by net, integrating or not the water column, can interfere in results (PINTO-CoELHo, 2004). Furthermore, the water volume for sampling should be enough to represent the environment diversity. For the aquatic environment, the standardization of sampling effort means that a minimal amount of collected water is established, per sample, for about $90 \%$ of the total environment richness to be registered (HECK et al., 1975).

Species accumulation curves are good tools for assessing the effectiveness of a sampling method, since they represent the cumulative number of species observed in an area (or volume) as a function of sampling effort (KING \& Porter, 2005; MuirheAd et al., 2006). On the other hand, rarefaction curves are used for direct comparisons between populations, or samples, to obtain the number of species expected in a random sample (MAGURRAN, 2011).

Due to seasonal variations in zooplankton composition and richness (Dumont \& Segers, 1996), a preliminary sampling is recommended to determine which sampling effort is required to access the highest possible richness in tropical aquatic environments, especially in temporary ponds. In the tropics, the high predation rates by planktivorous fish, cyanobacterial blooms and high pollution due to anthropogenic activities, are factors that influence the loss of diversity and have significant effects in water bodies (SARMA et al., 2005).

This study aims to determine the minimum water volume needed per sample collected for the largest possible number of zooplankton species in the limnetic region of Sapucaí River compartment of Furnas reservoir, and the sufficient number of monthly samples over a year to reach maximum richness.

\section{MATERIALS AND METHODS}

The reservoir of the Hydroelectric Power Plant (HPP) of Furnas is located in the south of the state of Minas Gerais, Brazil. It has a flooded area of $1,450 \mathrm{~km}^{2}$ and $250 \mathrm{~km}$ of length, in both of their two main sub-axes or compartments, the Sapucaí and Grande rivers, respectively (CoRGOSINHO \& Pinto-Coelho, 2006). The average depth is $13 \mathrm{~m}$ and the maximum reaches $90 \mathrm{~m}$, near the dam.

Samplings were carried out in HPP Furnas reservoir at the junction of rivers Verde and Sapucai (VSJ) $\left(21^{\circ} 27^{\prime} 03^{\prime \prime}\right.$ S and $45^{\circ} 40^{\prime} 24^{\prime \prime} \mathrm{W}$ ) in December 2009, with a plankton net of $68 \mu \mathrm{m}$ mesh size. First, seven samples of $100 \mathrm{~L}$ were collected sequentially integrating one-meter of water column, using a suction motor pump. After this, seven other samples of $70 \mathrm{~L}$ were collected with vertical hauls, using constant speed. Both methods were limited to onemeter depth from the water surface. The organisms were subjected to narcotization process with $\mathrm{CO}_{2}$ saturation by the addition of carbonated water to avoid body contraction of the zooplankton individuals. The samples were maintained in polyethylene bottles and fixed with formalin $4 \%(\mathrm{v} / \mathrm{v})$. Additional single samples of $400 \mathrm{~L}$ were collected monthly, in Barranco Alto region in this same reservoir, from March 2011 to February 2012. They were collected at stations BA1

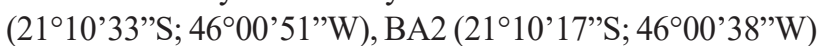
and BA3 $\left(21^{\circ} 10^{\prime} 04^{\prime \prime} \mathrm{S} ; 46^{\circ} 00^{\prime} 26^{\prime \prime} \mathrm{W}\right)$. The suction motor pump was used to integrate the water column, from the station depth to the water surface, and the sample was prepared and maintained as above.

The quantitative analysis, restricted to cladocerans and rotifers, was carried out on a gridded acrylic slide, under a stereoscope microscope (50x magnification), in sub-samples or the whole sample, depending on the sample characteristics. An optical microscope (up to 2000x magnification) and specialized bibliography were used for species identification (Koste, 1978; JersabeK et al., 2003; De Melo \& Hebert, 1994; ElMOOR-LOUREIRO, 1997; OrLOVA-BIENKOWSKAJA, 1998; SMirnov, 1998; SEgERs, 2007; Kotov, 2009; Sinev \& ELMOOR-LoureIro, 2010). The frequency of species was determined considering the number of samples in which they occurred in relation to the total number of samples. The species were classified as constant (present in more than $80 \%$ of samples), frequent (between 50 to $80 \%$ ), common (from 20 to 50\%) and rare $(<20 \%)$ (DAJOZ, 1983).

From the sample analysis the non-parametric estimators (ACE, Chao1, Jackknife1 and Bootstrap) of species richness were calculated based on abundance using the EstimateS 8.2 software (CoLwELL, 2009). Generally, these estimators make comparisons between rare species (uniques or singletons), present in a unique sample, and species present in, at least, two samples (doubletons or duplicates). These estimates were chosen according to KING \& PORTER (2005) and Sousa et al. (2014).

The richness evaluation was done by asymptotic behavior analysis of curves obtained from richness estimators.

\section{RESULTS}

Firstly, the analysis of samples from the VSJ station (unique sampling data) was carried out. The curves of ACE, Chao 1, Jackknife 1 and Bootstrap estimators, as well as the observed richness curve (Sobs), for the two types of samplings (vertical hauls with mesh and suction motor pump) both adjusted for increasing volume using the seven collected samples, in sequence, showed that $90 \%$ of the maximum richness was reached, on average, with up to $400 \mathrm{~L}$ of filtered water (see Figs 2, 3).

For the suction pump sampling, the asymptote was obtained by filtration of $600 \mathrm{~L}$. Only the Jackknife 1 estimator curve showed the organism richness increasing up to $700 \mathrm{~L}$ of sample, given that the curve of uniques began to decrease again (Fig. 2). For vertical haul sampling, about $80 \%$ of the maximum richness was reached using up to $140 \mathrm{~L}$ and above $95 \%$ using up to $350 \mathrm{~L}$, for every richness curve. The 

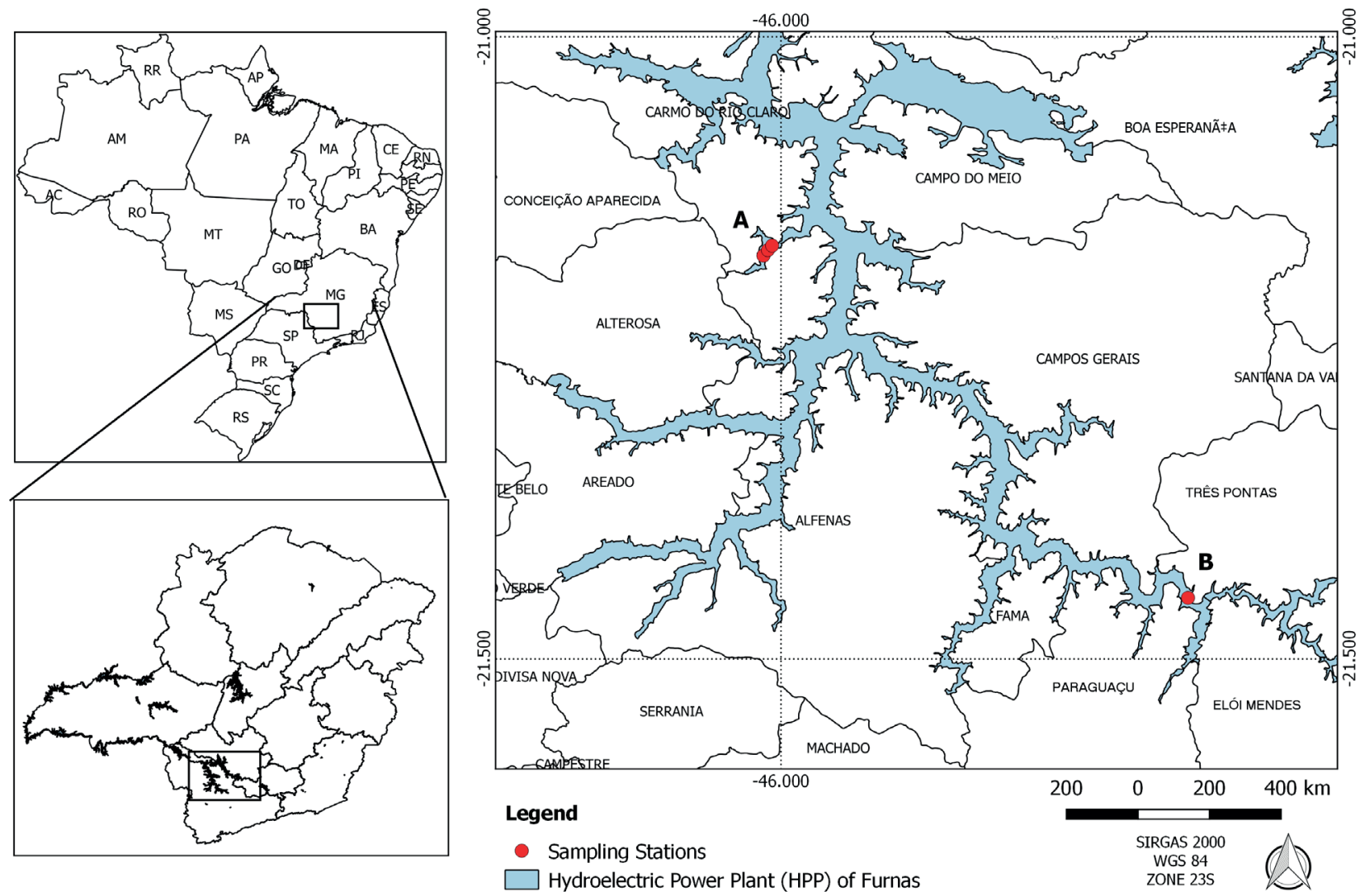

Fig 1. Sampling stations in the Hydroelectric Power Plant of Furnas reservoir, state of Minas Gerais, Brazil (A, Barranco Alto region; B, junction of rivers Verde and Sapucai - VSJ).

observation of estimator curves showed that the asymptote was reached using up to $420 \mathrm{~L}$ (Fig. 3).

Bosmina freyi, Bosminopsis deitersi, Moina minuta, Asplanchna sieboldi, Filinia longiseta, Hexarthra intermedia, Kellicotia bostoniensis and Synchaeta sp. were found for every one of seven samples which were collected with suction motor pump and vertical haul samplings. However, Ceriodaphnia cornuta rigaudi, Brachionus falcatus, Collotheca sp., Conochilus sp., Keratella americana, Lecane curvicornis, Platyas quadricornis, Plationus patulus, Polyarthra sp. and Testudinella patina were constant only in samplings using vertical hauls (Tabs I, II).

The rare species Tricocherca bicristata, Beucampiella sp., Chydorus eurynotus and Ascomorpha sp., as well as Lecane cornuta, Ptygura libera, Brachionus calyciflorus, Iliocryptus spinifer and Alonella lineolata were recorded in only one of the seven samples, being the earliest obtained from vertical hauls and the latest from suction pump sampling.

For analysis in time scale, twelve samples were collected every month for one year. Standard species accumulation curves were similar for the three sampling stations BA1, BA2 and BA3, showed by Figures 3, 5 and 6. Chao 1 and ACE estimator curves approximately overlapped with the Sobs curve, because there were no singletons or doubletons in the samples and species occurred in more than 10 ind. $\mathrm{L}^{-1}$. Species with low frequency (uniques and duplicates) were recorded at high density, so Jackknife 1 and Bootstrap estimator curves showed species richness above those of the Sobs curve, in all samples. The minimal sampling effort was reached, for all curves, on the $8^{\text {th }}$ sampling (asymptote), for both dry and wet seasons samplings.

Bosmina hagmanni, Ceriodaphnia cornuta cornuta, C. cornuta rigaudi, C. silvestrii, Daphnia gessneri, Diaphanosoma birgei, D. spinulosum, Moina minuta, Conochilus unicornis and Keratella americana were constant in all monthly samples (Tab. III).

\section{DISCUSSION}

The two tested sampling methods, suction pump and vertical hauls, showed similar results and included in the inventory most of the species already registered for this reservoir, thus the two sampling methods are comparable. At $400 \mathrm{~L}$ water volume about $90 \%$ of the environmental species had been accessed for samplings using suction pump and a similar percentage was recorded with $350 \mathrm{~L}$ for samplings with vertical hauls using plankton net. It is considered a satisfactory inventory of species when between 50 and $75 \%$ of the species which could potentially occur in the environment are registered and the more frequently found species (or common) should be included in this percentage (HECK et al., 1975).

The greater species richness was recorded for samplings using vertical hauls with a plankton net. Rotifers 


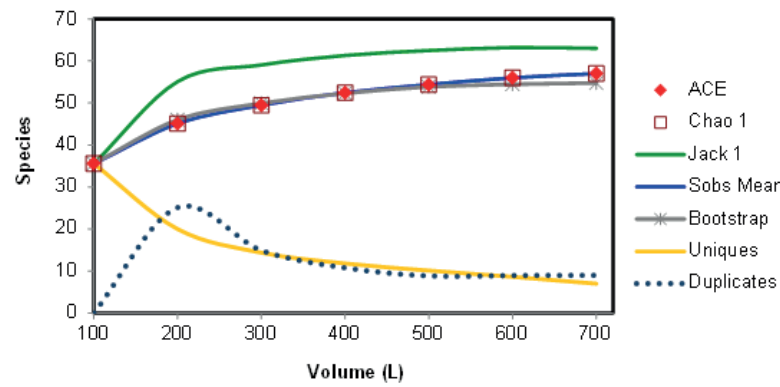

Fig. 2. Species accumulation curves, uniques and duplicates for the VSJ station in Furnas reservoir, state of Minas Gerais, Brazil, collected by suction pump.

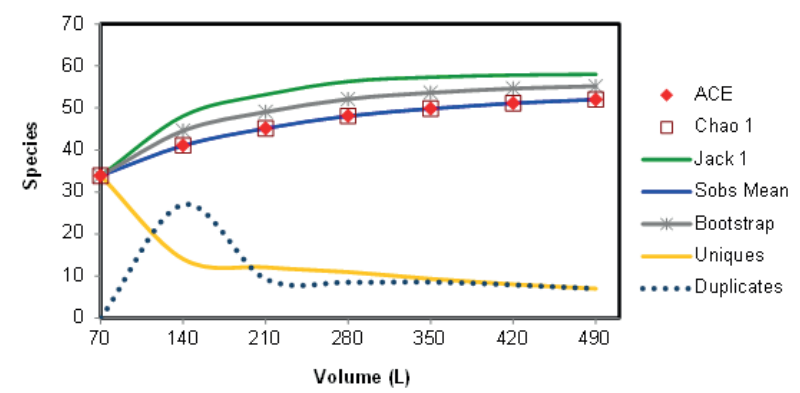

Fig. 3. Species accumulation curves, uniques and duplicates for the VSJ station in Furnas reservoir, state of Minas Gerais, Brazil, collected with vertical hauls.

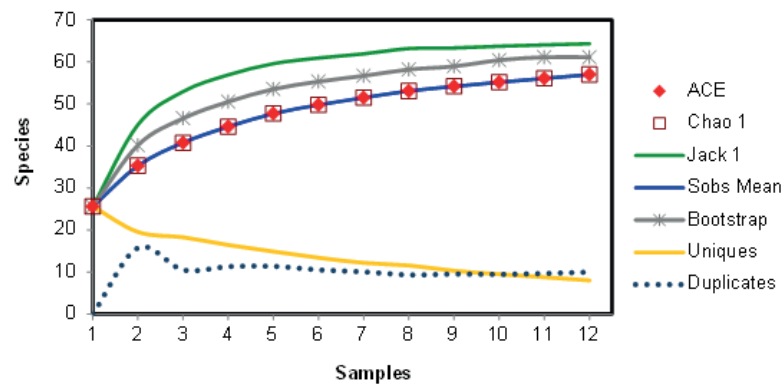

Fig. 4. Species accumulation curves, uniques and duplicates for the BA1 station of Furnas reservoir, state of Minas Gerais, Brazil, from March 2011 to February 2012.

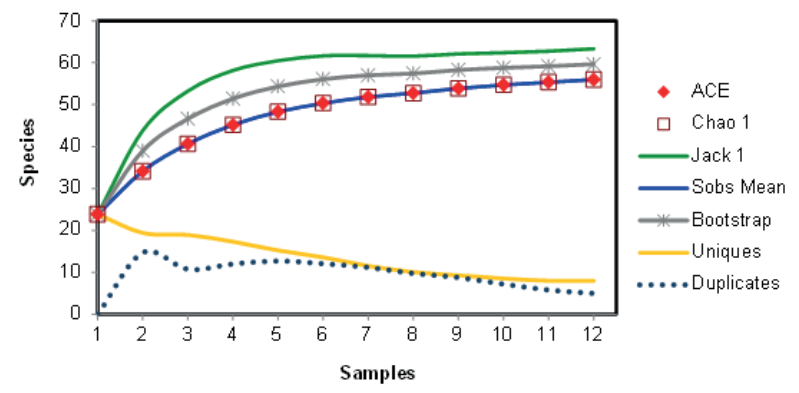

Fig. 5. Species accumulation curves, uniques and duplicates for the BA2 station of Furnas reservoir, state of Minas Gerais, Brazil from March 2011 to February 2012.

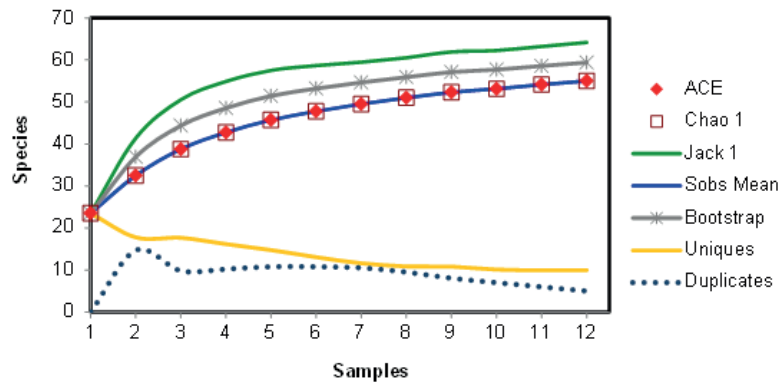

Fig. 6. Species accumulation curves, uniques and duplicates for the BA3 station of Furnas reservoir, state of Minas Gerais from March 2011 to February 2012.

were the most specious in this type of sampling while cladocerans were more representative with suction motor pump samplings. The most sensitive organisms, such as rotifers, may be damaged (including breakup and destruction of some individuals) during suction pump samplings, which makes their identification difficult (KoZLOWSKY-SUZUKI \& Bozelli, 1998; Pinto-Coelho, 2004). On the other hand, the suction motor pump samplings select organisms with reduced escape capability and low (and slow) swimming movements, such as Cladocera (Pinto-CoelHo, 2004).

The species richness for the three stations was very similar regarding the sampling throughout the year. With increasing sampling effort, the rarest species were identified. However, when the sampling effort is excessive, errant species are recorded, contributing to an increase in the uniques curve and even in this case the estimators extrapolate the total richness of the environment (MAGURRAN, 2011). Thus, it is believed that in 20 rare species sampled; only $8 \%$ are truly rare (KING \& PORTER, 2005).

Many of the species identified in other studies in the Furnas reservoir were not recorded in this study, as they covered a larger area of this reservoir surrounding the limnetic and littoral regions and the two compartments (Rivers Grande and Sapucaí), resulting in greater richness. In the review by SANTOS-WISNIEWSKI et al. (2011) for Cladocera fauna of Minas Gerais, 94 species were recorded for the state distributed in 88 water bodies. Of these, 62 species were identified in the Furnas reservoir. Among the species not recorded in this study in the limnetic region were Bosmina longirostris, Bosmina tubicen, Daphnia ambigua, Simocephalus latirostris, Simocephalus serrulatus and Moina micrura.

Generally, the records of Bosmina longirostris for Brazil are dubious, because according to DE MELO \& HEBERT (1994), the species occurs in North America and B. freyi in South America. In the present study and in earlier studies in the Furnas reservoir, only B. freyi was recorded.

Although the study was carried out in the limnetic region, typical species of the littoral region were recorded, such as Alona intermedia, Alona yara, Alona guttata, Camptocercus australis, Chydorus pubescens, Chydorus eurynotus, Ephemeroporus sp., Ilyocryptus spinifer and 
Tab. I. List of occurrence of zooplankton species and Dajoz Constancy Index (DCI) for the seven samples (S) collected by suction pump at the VSJ station in Furnas reservoir, state of Minas Gerais, Brazil (Ct, constants; F, frequent; C, common; R, rare).

\begin{tabular}{|c|c|c|c|c|c|c|c|c|c|}
\hline Taxa & $\mathrm{S} 1$ & $\mathrm{~S} 2$ & S3 & S4 & S5 & S6 & S7 & & \\
\hline CLADOCERA & & & & & & & & $\%$ & DCI \\
\hline Alona sp. & $\mathrm{X}$ & $\mathrm{X}$ & & & & & $\mathrm{X}$ & 43 & $\mathrm{C}$ \\
\hline Alonella lineolata Sars, 1901 & & & $\mathrm{X}$ & & & & & 14 & $\mathrm{R}$ \\
\hline Bosmina freyi De Melo \& Hebert, 1994 & $\mathrm{X}$ & $\mathrm{X}$ & $\mathrm{X}$ & $\mathrm{X}$ & $\mathrm{X}$ & $\mathrm{X}$ & $\mathrm{X}$ & 100 & $\mathrm{Ct}$ \\
\hline Bosmina hagmanni Stingelin, 1904 & & & & $\mathrm{X}$ & $\mathrm{X}$ & $\mathrm{X}$ & & 43 & $\mathrm{C}$ \\
\hline Bosminopsis deitersi Richard, 1895 & $\mathrm{X}$ & $\mathrm{X}$ & $\mathrm{X}$ & $\mathrm{X}$ & $\mathrm{X}$ & $\mathrm{X}$ & $\mathrm{X}$ & 100 & $\mathrm{Ct}$ \\
\hline Ceriodaphnia cornuta Sars, 1886 & & $\mathrm{X}$ & & & & & & 14 & $\mathrm{R}$ \\
\hline Ceriodaphnia cornuta cornuta Sars, 1886 & & & $\mathrm{X}$ & & $\mathrm{X}$ & $\mathrm{X}$ & $\mathrm{X}$ & 57 & $\mathrm{~F}$ \\
\hline Ceriodaphnia cornuta intermedia Sars, 1886 & $\mathrm{X}$ & & & & & & $\mathrm{X}$ & 29 & $\mathrm{C}$ \\
\hline Ceriodaphnia cornuta rigaudi Sars, 1886 & $\mathrm{X}$ & & $\mathrm{X}$ & $\mathrm{X}$ & $\mathrm{X}$ & $\mathrm{X}$ & & 71 & $\mathrm{~F}$ \\
\hline Ceriodaphnia silvestrii Daday, 1902 & $\mathrm{X}$ & & $\mathrm{X}$ & $\mathrm{X}$ & $\mathrm{X}$ & $\mathrm{X}$ & & 71 & $\mathrm{~F}$ \\
\hline Daphnia gessneri Herbest, 1967 & & $\mathrm{X}$ & & $\mathrm{X}$ & $\mathrm{X}$ & $\mathrm{X}$ & & 57 & $\mathrm{~F}$ \\
\hline Diaphanosoma birgei Korineck, 1981 & $\mathrm{X}$ & $\mathrm{X}$ & & $\mathrm{X}$ & $\mathrm{X}$ & $\mathrm{X}$ & $\mathrm{X}$ & 86 & $\mathrm{Ct}$ \\
\hline Diaphanosoma spinulosum Korineck, 1981 & $\mathrm{X}$ & & $\mathrm{X}$ & $\mathrm{X}$ & $\mathrm{X}$ & $\mathrm{X}$ & $\mathrm{X}$ & 86 & $\mathrm{Ct}$ \\
\hline Diaphanosoma sp. & $\mathrm{X}$ & & $\mathrm{X}$ & & & $\mathrm{X}$ & & 43 & $\mathrm{C}$ \\
\hline Ilyocryptus spinifer Korineck, 1981 & & & & & & $\mathrm{X}$ & & 14 & $\mathrm{R}$ \\
\hline Leydigia striata Berabén, 1939 & & & $\mathrm{X}$ & $\mathrm{X}$ & & & & 29 & $\mathrm{C}$ \\
\hline Macrothrix sp. & $\mathrm{X}$ & $\mathrm{X}$ & $\mathrm{X}$ & $\mathrm{X}$ & & $\mathrm{X}$ & $\mathrm{X}$ & 86 & $\mathrm{Ct}$ \\
\hline Moina minuta Hansen, 1899 & $\mathrm{X}$ & $\mathrm{X}$ & $\mathrm{X}$ & $\mathrm{X}$ & $\mathrm{X}$ & $\mathrm{X}$ & $\mathrm{X}$ & 100 & $\mathrm{Ct}$ \\
\hline ROTIFERA & & & & & & & & 0 & \\
\hline Asplanchna sieboldi (Leydig, 1854) & $\mathrm{X}$ & $\mathrm{X}$ & $\mathrm{X}$ & $\mathrm{X}$ & $\mathrm{X}$ & $\mathrm{X}$ & $\mathrm{X}$ & 100 & $\mathrm{Ct}$ \\
\hline Beucampiella sp. & $\mathrm{X}$ & & $\mathrm{X}$ & $\mathrm{X}$ & $\mathrm{X}$ & $\mathrm{X}$ & $\mathrm{X}$ & 86 & $\mathrm{Ct}$ \\
\hline Brachionus calyciflorus Pallas, 1766 & & & & $\mathrm{X}$ & & & & 14 & $\mathrm{R}$ \\
\hline Brachionus dolabratus Harring, 1915 & & & & $\mathrm{X}$ & & $\mathrm{X}$ & $\mathrm{X}$ & 43 & $\mathrm{C}$ \\
\hline Brachionus falcatus Zacharias, 1898 & & $\mathrm{X}$ & $\mathrm{X}$ & $\mathrm{X}$ & $\mathrm{X}$ & & $\mathrm{X}$ & 71 & $\mathrm{~F}$ \\
\hline Brachionus mirus (Daday, 1905) & $\mathrm{X}$ & $\mathrm{X}$ & & & & & $\mathrm{X}$ & 43 & $\mathrm{C}$ \\
\hline Collotheca sp. & & $\mathrm{X}$ & & $\mathrm{X}$ & $\mathrm{X}$ & $\mathrm{X}$ & $\mathrm{X}$ & 71 & $\mathrm{~F}$ \\
\hline Conochilus sp. & & & & $\mathrm{X}$ & $\mathrm{X}$ & $\mathrm{X}$ & $\mathrm{X}$ & 57 & $\mathrm{~F}$ \\
\hline Conochilus unicornis (Rousselet, 1892) & & $\mathrm{X}$ & & & & $\mathrm{X}$ & & 29 & $\mathrm{C}$ \\
\hline Euchlanis dilatata Ehrenber, 1832 & & $\mathrm{X}$ & $\mathrm{X}$ & & & & & 29 & $\mathrm{C}$ \\
\hline Filinia longiseta (Ehrenberg, 1834) & $\mathrm{X}$ & $\mathrm{X}$ & $\mathrm{X}$ & $\mathrm{X}$ & $\mathrm{X}$ & $\mathrm{X}$ & $\mathrm{X}$ & 100 & $\mathrm{Ct}$ \\
\hline Filinia opoliensis (Zacharias, 1898) & & & & $\mathrm{X}$ & & $\mathrm{X}$ & & 29 & $\mathrm{C}$ \\
\hline Hexartra intermedia (Weiszniewski, 1929) & $\mathrm{X}$ & $\mathrm{X}$ & $\mathrm{X}$ & $\mathrm{X}$ & $\mathrm{X}$ & $\mathrm{X}$ & $\mathrm{X}$ & 100 & $\mathrm{Ct}$ \\
\hline Kellicotia bostoniensis (Rousselete, 1908) & $\mathrm{X}$ & $\mathrm{X}$ & $\mathrm{X}$ & $\mathrm{X}$ & $\mathrm{X}$ & $\mathrm{X}$ & $\mathrm{X}$ & 100 & $\mathrm{Ct}$ \\
\hline Keratella americana (Carlin, 1943) & & $\mathrm{X}$ & $\mathrm{X}$ & $\mathrm{X}$ & $\mathrm{X}$ & $\mathrm{X}$ & $\mathrm{X}$ & 86 & $\mathrm{Ct}$ \\
\hline Keratella cochlearis (Gosse, 1851) & $\mathrm{X}$ & $\mathrm{X}$ & & $\mathrm{X}$ & $\mathrm{X}$ & & $\mathrm{X}$ & 71 & $\mathrm{~F}$ \\
\hline Keratella lenzi (Hauer, 1953) & & $\mathrm{X}$ & $\mathrm{X}$ & & & & & 29 & $\mathrm{C}$ \\
\hline Keratella tropica (Apstein 1907) & & $\mathrm{X}$ & $\mathrm{X}$ & & $\mathrm{X}$ & & $\mathrm{X}$ & 57 & $\mathrm{~F}$ \\
\hline Lecane bulla (Gosse, 1886) & & & & $\mathrm{X}$ & & $\mathrm{X}$ & & 29 & $\mathrm{C}$ \\
\hline Lecane cornuta (Müller, 1786) & & $\mathrm{X}$ & & & & & & 14 & $\mathrm{R}$ \\
\hline Lecane curvicornis (Murray, 1913) & $\mathrm{X}$ & $\mathrm{X}$ & & $\mathrm{X}$ & $\mathrm{X}$ & $\mathrm{X}$ & $\mathrm{X}$ & 86 & $\mathrm{Ct}$ \\
\hline Lecane proiecta Hauer, 1956 & $\mathrm{X}$ & $\mathrm{X}$ & $\mathrm{X}$ & $\mathrm{X}$ & & $\mathrm{X}$ & & 71 & $\mathrm{~F}$ \\
\hline Lepadella sp. & & & $\mathrm{X}$ & $\mathrm{X}$ & & $\mathrm{X}$ & & 43 & $\mathrm{C}$ \\
\hline Platyas quadricornis (Ehrenberg, 1832) & $\mathrm{X}$ & $\mathrm{X}$ & $\mathrm{X}$ & & $\mathrm{X}$ & $\mathrm{X}$ & $\mathrm{X}$ & 86 & $\mathrm{Ct}$ \\
\hline Plationus patulus (Müller, 1953) & $\mathrm{X}$ & $\mathrm{X}$ & $\mathrm{X}$ & $\mathrm{X}$ & $\mathrm{X}$ & & $\mathrm{X}$ & 86 & $\mathrm{Ct}$ \\
\hline Polyarthra sp. & $\mathrm{X}$ & $\mathrm{X}$ & $\mathrm{X}$ & & $\mathrm{X}$ & & $\mathrm{X}$ & 71 & $\mathrm{~F}$ \\
\hline Proales sp. & $\mathrm{X}$ & $\mathrm{X}$ & $\mathrm{X}$ & $\mathrm{X}$ & $\mathrm{X}$ & $\mathrm{X}$ & & 86 & $\mathrm{Ct}$ \\
\hline Ptygura libera Myers, 1934 & & & & & & & $\mathrm{X}$ & 14 & $\mathrm{R}$ \\
\hline Sinchaeta $\mathrm{sp}$ & $\mathrm{X}$ & $\mathrm{X}$ & $\mathrm{X}$ & $\mathrm{X}$ & $\mathrm{X}$ & $\mathrm{X}$ & $\mathrm{X}$ & 100 & $\mathrm{Ct}$ \\
\hline Testudinella patina (Hermann, 1783) & $\mathrm{X}$ & $\mathrm{X}$ & X & & X & & X & 71 & $\mathrm{~F}$ \\
\hline Trichocerca cylindrica (Sudzuki 1956) & & & & $\mathrm{X}$ & $\mathrm{X}$ & & $\mathrm{X}$ & 43 & $\mathrm{C}$ \\
\hline
\end{tabular}

Tab. II. List of occurrence of zooplankton species and Dajoz Constancy Index (DCI) for the seven samples (S) collected with vertical hauls in VSJ station in Furnas reservoir, state of Minas Gerais, Brazil. (Ct, constants; F, frequent; C, common; R, rare).

\begin{tabular}{|c|c|c|c|c|c|c|c|c|c|}
\hline Taxa & $\mathrm{S} 1$ & $\mathrm{~S} 2$ & S3 & S4 & S5 & S6 & $\mathrm{S} 7$ & & \\
\hline CLADOCERA & & & & & & & & $\%$ & DCI \\
\hline Alona sp. & & $\mathrm{X}$ & & & & & & 14 & $\mathrm{R}$ \\
\hline Alonella dadayi Birgei, 1910 & & $\mathrm{X}$ & $\mathrm{X}$ & & & & & 29 & $\mathrm{C}$ \\
\hline Bosmina freyi De Melo \& Hebert, 1994 & $\mathrm{X}$ & $\mathrm{X}$ & $\mathrm{X}$ & $\mathrm{X}$ & $\mathrm{X}$ & $\mathrm{X}$ & $\mathrm{X}$ & 100 & $\mathrm{Ct}$ \\
\hline Bosmina hagmanni Stingelin, 1904 & $\mathrm{X}$ & $\mathrm{X}$ & & $\mathrm{X}$ & & $\mathrm{X}$ & $\mathrm{X}$ & 71 & $\mathrm{~F}$ \\
\hline Bosminopsis deitersi Richard, 1895 & $\mathrm{X}$ & $\mathrm{X}$ & $\mathrm{X}$ & $\mathrm{X}$ & $\mathrm{X}$ & $\mathrm{X}$ & $\mathrm{X}$ & 100 & $\mathrm{Ct}$ \\
\hline Ceriodaphnia cornuta cornuta Sars, 1886 & $\mathrm{X}$ & $\mathrm{X}$ & $\mathrm{X}$ & $\mathrm{X}$ & $\mathrm{X}$ & & $\mathrm{X}$ & 86 & $\mathrm{Ct}$ \\
\hline Ceriodaphnia cornuta rigaudi Sars, 1886 & $\mathrm{X}$ & $\mathrm{X}$ & $\mathrm{X}$ & $\mathrm{X}$ & $\mathrm{X}$ & $\mathrm{X}$ & $\mathrm{X}$ & 100 & $\mathrm{Ct}$ \\
\hline Ceriodaphnia silvestrii Daday, 1902 & $\mathrm{X}$ & $\mathrm{X}$ & & & & $\mathrm{X}$ & $\mathrm{X}$ & 57 & $\mathrm{~F}$ \\
\hline
\end{tabular}


Tab. II. Cont.

\begin{tabular}{|c|c|c|c|c|c|c|c|c|c|}
\hline Taxa & S1 & S2 & S3 & S4 & S5 & S6 & S7 & & \\
\hline Chydorus eurynotus Sars, 1901 & & & $\mathrm{X}$ & & & & & 14 & $\mathrm{R}$ \\
\hline Daphnia gessneri Herbest, 1967 & & & & $\mathrm{X}$ & & $\mathrm{X}$ & $\mathrm{X}$ & 43 & $\mathrm{C}$ \\
\hline Diaphanosoma spinulosum Korineck, 1981 & $\mathrm{X}$ & $\mathrm{X}$ & $\mathrm{X}$ & $\mathrm{X}$ & $\mathrm{X}$ & $X$ & & 86 & $\mathrm{Ct}$ \\
\hline Diaphanosoma sp. & $\mathrm{X}$ & $\mathrm{X}$ & $\mathrm{X}$ & $\mathrm{X}$ & $\mathrm{X}$ & $\mathrm{X}$ & $\mathrm{X}$ & 100 & $\mathrm{Ct}$ \\
\hline Macrothrix sp. & $\mathrm{X}$ & & & $\mathrm{X}$ & $\mathrm{X}$ & $\mathrm{X}$ & & 57 & $\mathrm{~F}$ \\
\hline Moina minuta Hansen, 1899 & $\mathrm{X}$ & $\mathrm{X}$ & $\mathrm{X}$ & $\mathrm{X}$ & $\mathrm{X}$ & $\mathrm{X}$ & $\mathrm{X}$ & 100 & $\mathrm{Ct}$ \\
\hline ROTIFERA & & $\mathrm{X}$ & & & & & & 14 & $\mathrm{R}$ \\
\hline Ascomorpha sp. & & & & & & & & & \\
\hline Asplanchna sieboldi (Leydig, 1854) & $\mathrm{X}$ & $\mathrm{X}$ & $\mathrm{X}$ & $\mathrm{X}$ & $\mathrm{X}$ & $\mathrm{X}$ & $\mathrm{X}$ & 100 & $\mathrm{Ct}$ \\
\hline Beucampiella sp. & $\mathrm{X}$ & & & & & & & 14 & $\mathrm{R}$ \\
\hline Brachionus calyciflorus Pallas, 1766 & & & $\mathrm{X}$ & & & & $\mathrm{X}$ & 29 & $\mathrm{C}$ \\
\hline Brachionus dolabratus Harring, 1915 & & $\mathrm{X}$ & $\mathrm{X}$ & $\mathrm{X}$ & & & & 43 & $\mathrm{C}$ \\
\hline Brachionus falcatus Zacharias, 1898 & $\mathrm{X}$ & $\mathrm{X}$ & $\mathrm{X}$ & $\mathrm{X}$ & $\mathrm{X}$ & $\mathrm{X}$ & $\mathrm{X}$ & 100 & $\mathrm{Ct}$ \\
\hline Brachionus mirus (Daday, 1905) & & & & & $\mathrm{X}$ & $\mathrm{X}$ & $\mathrm{X}$ & 43 & $\mathrm{C}$ \\
\hline Cephalodella sp. & & & & $\mathrm{X}$ & $\mathrm{X}$ & & & 29 & $\mathrm{C}$ \\
\hline Collotheca sp. & $\mathrm{X}$ & $\mathrm{X}$ & $\mathrm{X}$ & $\mathrm{X}$ & $\mathrm{X}$ & $\mathrm{X}$ & $\mathrm{X}$ & 100 & $\mathrm{Ct}$ \\
\hline Conochilus sp. & $\mathrm{X}$ & $\mathrm{X}$ & $\mathrm{X}$ & $\mathrm{X}$ & $\mathrm{X}$ & $\mathrm{X}$ & $\mathrm{X}$ & 100 & $\mathrm{Ct}$ \\
\hline Conochilus coenobasis (Skorikov, 1914) & & $\mathrm{X}$ & & & & & & 14 & $\mathrm{R}$ \\
\hline Conochilus unicornis (Rousselet, 1892) & $\mathrm{X}$ & $\mathrm{X}$ & & & & & & 29 & $\mathrm{C}$ \\
\hline Euchlanis dilatata Ehrenber, 1832 & & $\mathrm{X}$ & & & $\mathrm{X}$ & & $\mathrm{X}$ & 43 & $\mathrm{C}$ \\
\hline Filinia longiseta (Ehrenberg, 1834) & $\mathrm{X}$ & $\mathrm{X}$ & $\mathrm{X}$ & $\mathrm{X}$ & $\mathrm{X}$ & $\mathrm{X}$ & $\mathrm{X}$ & 100 & $\mathrm{Ct}$ \\
\hline Filinia opoliensis (Zacharias, 1898) & & & & & $\mathrm{X}$ & $\mathrm{X}$ & $\mathrm{X}$ & 43 & $\mathrm{C}$ \\
\hline Hexartra intermedia (Weiszniewski, 1929) & $\mathrm{X}$ & $\mathrm{X}$ & $\mathrm{X}$ & $\mathrm{X}$ & $\mathrm{X}$ & $\mathrm{X}$ & $\mathrm{X}$ & 100 & $\mathrm{Ct}$ \\
\hline Kellicotia bostoniensis (Rousselete, 1908) & $\mathrm{X}$ & $\mathrm{X}$ & $\mathrm{X}$ & $\mathrm{X}$ & $\mathrm{X}$ & $\mathrm{X}$ & $\mathrm{X}$ & 100 & $\mathrm{Ct}$ \\
\hline Keratella americana (Carlin, 1943) & $\mathrm{X}$ & $\mathrm{X}$ & $\mathrm{X}$ & $\mathrm{X}$ & $\mathrm{X}$ & $\mathrm{X}$ & $\mathrm{X}$ & 100 & $\mathrm{Ct}$ \\
\hline Keratella cochlearis (Gosse, 1851) & $\mathrm{X}$ & & $\mathrm{X}$ & $\mathrm{X}$ & $\mathrm{X}$ & $\mathrm{X}$ & $\mathrm{X}$ & 86 & $\mathrm{Ct}$ \\
\hline Keratella lenzi (Hauer, 1953) & $\mathrm{X}$ & $\mathrm{X}$ & $\mathrm{X}$ & & $\mathrm{X}$ & $\mathrm{X}$ & $\mathrm{X}$ & 86 & $\mathrm{Ct}$ \\
\hline Keratella tropica (Apstein 1907) & & & $\mathrm{X}$ & & $\mathrm{X}$ & $\mathrm{X}$ & $\mathrm{X}$ & 57 & $\mathrm{~F}$ \\
\hline Lecane bulla (Gosse, 1886) & $\mathrm{X}$ & & $\mathrm{X}$ & & & $\mathrm{X}$ & $\mathrm{X}$ & 57 & $\mathrm{~F}$ \\
\hline Lecane curvicornis (Murray, 1913) & $\mathrm{X}$ & $\mathrm{X}$ & $\mathrm{X}$ & $\mathrm{X}$ & $\mathrm{X}$ & $\mathrm{X}$ & $\mathrm{X}$ & 100 & $\mathrm{Ct}$ \\
\hline Lecane leontina (Turner, 1892) & $\mathrm{X}$ & $\mathrm{X}$ & $\mathrm{X}$ & $\mathrm{X}$ & $\mathrm{X}$ & & & 71 & $\mathrm{~F}$ \\
\hline Lecane papuana Murray 1913 & & $\mathrm{X}$ & $\mathrm{X}$ & $\mathrm{X}$ & & & & 43 & $\mathrm{C}$ \\
\hline Lecane proiecta Hauer, 1956 & & & $\mathrm{X}$ & & & $\mathrm{X}$ & & 29 & $\mathrm{~F}$ \\
\hline Lecane quadridentata (Ehrenberg, 1832) & & & $\mathrm{X}$ & $\mathrm{X}$ & & & & 29 & $\mathrm{~F}$ \\
\hline Lecane sp. & & & $\mathrm{X}$ & & & & & 14 & $\mathrm{R}$ \\
\hline Platyas quadricornis (Ehrenberg, 1832) & $\mathrm{X}$ & $\mathrm{X}$ & $\mathrm{X}$ & $\mathrm{X}$ & $\mathrm{X}$ & $\mathrm{X}$ & $\mathrm{X}$ & 100 & $\mathrm{Ct}$ \\
\hline Plationus macracanthus (Daday, 1905) & $\mathrm{X}$ & $\mathrm{X}$ & $\mathrm{X}$ & $\mathrm{X}$ & $\mathrm{X}$ & $\mathrm{X}$ & & 86 & $\mathrm{Ct}$ \\
\hline Plationus patulus (Müller, 1953) & $\mathrm{X}$ & $\mathrm{X}$ & $\mathrm{X}$ & $\mathrm{X}$ & $\mathrm{X}$ & $\mathrm{X}$ & $\mathrm{X}$ & 100 & $\mathrm{Ct}$ \\
\hline Polyarthra sp. & $\mathrm{X}$ & $\mathrm{X}$ & $\mathrm{X}$ & $\mathrm{X}$ & $\mathrm{X}$ & $\mathrm{X}$ & $\mathrm{X}$ & 100 & $\mathrm{Ct}$ \\
\hline Proales sp. & $\mathrm{X}$ & $\mathrm{X}$ & $\mathrm{X}$ & $\mathrm{X}$ & $\mathrm{X}$ & $\mathrm{X}$ & & 86 & $\mathrm{Ct}$ \\
\hline Synchaeta sp. & $\mathrm{X}$ & $\mathrm{X}$ & $\mathrm{X}$ & $\mathrm{X}$ & $\mathrm{X}$ & $\mathrm{X}$ & $\mathrm{X}$ & 100 & $\mathrm{Ct}$ \\
\hline Testudinella patina (Hermann, 1783) & $\mathrm{X}$ & $\mathrm{X}$ & $\mathrm{X}$ & $\mathrm{X}$ & $\mathrm{X}$ & $\mathrm{X}$ & $\mathrm{X}$ & 100 & $\mathrm{Ct}$ \\
\hline Trichocerca bicristata (Gosse, 1887) & & & & $\mathrm{X}$ & & & & 14 & $\mathrm{R}$ \\
\hline Trichocerca cylindrica (Suzuki 1956) & & & & & $\mathrm{X}$ & & $\mathrm{X}$ & 29 & $\mathrm{C}$ \\
\hline Trichotria tetractis (Ehrenberg, 1830) & & $\mathrm{X}$ & & & $\mathrm{X}$ & $\mathrm{X}$ & & 43 & $\mathrm{~F}$ \\
\hline
\end{tabular}

Tab. III. List of occurrence of zooplankton species and Dajoz Constancy Index (DCI) in site BA1, BA2 and BA3 stations, from March 2011 to February 2012 (Ct, constants; F, frequent; C, common; R, rare;*, occurrence in 1 station; **, occurrence in 2 station; ***, occurrence in 3 stations).

\begin{tabular}{|c|c|c|c|c|c|c|c|c|c|c|c|c|c|c|}
\hline Taxa & Mar & Apr & May & Jun & Jul & Aug & Sep & Oct & Nov & Dec & Jan & Feb & $\%$ & ICD \\
\hline \multicolumn{15}{|l|}{ CLADOCERA } \\
\hline Alona guttata Sars, 1862 & & & & $*$ & $*$ & $* * *$ & $*$ & $*$ & & $*$ & & $*$ & 25 & $\mathrm{c}$ \\
\hline Alona yara Sinev \& Elmoor-Loureiro, 2010 & & & & & & & & & $* *$ & $*$ & $* *$ & $*$ & 17 & $\mathrm{R}$ \\
\hline Alona intermedia Sars, 1862 & & & $* *$ & & & & & & & & & & 6 & $\mathrm{R}$ \\
\hline Bosmina freyi De Melo \& Hebert, 1994 & $* *$ & & $* * *$ & $* * *$ & $* * *$ & $* * *$ & $* * *$ & $* *$ & $* * *$ & $* * *$ & $* *$ & $* * *$ & 83 & $\mathrm{Ct}$ \\
\hline Bosmina hagmanni Stingelin, 1904 & $* * *$ & $* * *$ & $* * *$ & $* * *$ & $* * *$ & $* * *$ & $* * *$ & $* * *$ & $* * *$ & $* * *$ & $* * *$ & $* * *$ & 100 & $\mathrm{Ct}$ \\
\hline Camptochercus australis Sars, 1896 & & & & & & & & & & & $*$ & & 3 & $\mathrm{R}$ \\
\hline Ceriodaphnia cornuta cornuta Sars, 1886 & $* * *$ & $* * *$ & $* * *$ & $* * *$ & $* * *$ & $* * *$ & $* * *$ & $* * *$ & $* * *$ & $* * *$ & $* *$ & $* * *$ & 97 & $\mathrm{Ct}$ \\
\hline Ceriodaphnia cornuta intermedia Sars, 1886 & & $*$ & $* *$ & $* *$ & $*$ & $*$ & & & $* * *$ & $* * *$ & $*$ & $* * *$ & 47 & $\mathrm{c}$ \\
\hline Ceriodaphnia cornuta rigaudi Sars, 1886 & $* * *$ & $* * *$ & $* * *$ & $* * *$ & $* * *$ & $* * *$ & $* * *$ & $* * *$ & $* * *$ & $* * *$ & $* * *$ & $* * *$ & 100 & $\mathrm{Ct}$ \\
\hline Ceriodaphnia silvestrii Daday, 1902 & $* * *$ & $* * *$ & $* * *$ & $* * *$ & $* * *$ & $* * *$ & $* * *$ & $* * *$ & $* * *$ & $* * *$ & $* * *$ & $* *$ & 97 & $\mathrm{Ct}$ \\
\hline Daphnia gessneri Herbest, 1967 & $* *$ & $* * *$ & $* *$ & $* * *$ & $* * *$ & $* *$ & $* * *$ & $* * *$ & $* * *$ & * & $* *$ & $* * *$ & 86 & $\mathrm{Ct}$ \\
\hline Daphnia laevis Birge, 1878 & & $*$ & & & & & $* *$ & $* * *$ & & & & & 17 & $\mathrm{R}$ \\
\hline Diaphanosoma birgei Korineck, 1981 & $* * *$ & $* * *$ & $* * *$ & $* * *$ & $* * *$ & $* *$ & $* *$ & $* * *$ & $* * *$ & $* * *$ & $* * *$ & $* * *$ & 94 & $\mathrm{Ct}$ \\
\hline Diaphanosoma brevireme Sars, 1901 & $* * *$ & $* *$ & $* *$ & & & $* *$ & $* *$ & $* * *$ & $* *$ & $* *$ & $* *$ & $* * *$ & 64 & $\mathrm{~F}$ \\
\hline
\end{tabular}


Tab. III. Cont.

\begin{tabular}{|c|c|c|c|c|c|c|c|c|c|c|c|c|c|c|}
\hline Taxa & Mar & Apr & May & Jun & Jul & Aug & Sep & Oct & Nov & Dec & Jan & Feb & $\%$ & ICD \\
\hline Diaphanosoma spinulosum Herbst, 1967 & $* * *$ & $* * *$ & $* * *$ & $* *$ & $* * *$ & $* *$ & $* *$ & $* *$ & $* * *$ & $* * *$ & $* * *$ & $* * *$ & 89 & $\mathrm{Ct}$ \\
\hline Diaphanosoma sp. & & * & $*$ & * & $*$ & $*$ & $*$ & & $*$ & $*$ & & $*$ & 25 & $\mathrm{c}$ \\
\hline Diaphanosoma fluviatile Hansen, 1899 & $*$ & $* *$ & & & $* *$ & $* *$ & $* *$ & & $* * *$ & $* * *$ & $* * *$ & $* * *$ & 58 & $\mathrm{~F}$ \\
\hline Ilyocryptus spinifer Herrick, 1882 & & $*$ & & $*$ & & & & & & & & & 6 & $\mathrm{R}$ \\
\hline Macrothrix sp. & & & & & & & $* *$ & $*$ & & $* *$ & & & 14 & $\mathrm{R}$ \\
\hline Moina minuta Hansen, 1899 & $* * *$ & $* * *$ & $* * *$ & $* * *$ & $* * *$ & $* * *$ & $* * *$ & $* * *$ & $* * *$ & $* * *$ & $* * *$ & $* * *$ & 100 & $\mathrm{Ct}$ \\
\hline Simocephalus mixtus Orlova-Bienkowskaja, 2001 & & & $*$ & $* *$ & & $* *$ & $* * *$ & $*$ & * & & & & 28 & $\mathrm{c}$ \\
\hline Chydorus pubescens Sars, 1901 & & & $*$ & $*$ & & $* * *$ & * & $* *$ & & & * & & 25 & $\mathrm{c}$ \\
\hline Chydorus sp. & & & & & & & & & * & & & & 3 & $\mathrm{R}$ \\
\hline \multicolumn{15}{|l|}{ ROTIFERA } \\
\hline Anuraeopsis sp. & & * & & & & & & & * & & & * & 8 & $\mathrm{R}$ \\
\hline Ascomorpha saltans Bartsch, 1870 & & & $*$ & & $* *$ & & $*$ & & $* *$ & $*$ & $*$ & & 22 & $\mathrm{c}$ \\
\hline Ascomorpha ovalis (Bergendal, 1892) & & & $* *$ & $*$ & $*$ & $* * *$ & $* * *$ & $* * *$ & $* *$ & $*$ & $*$ & $* *$ & 53 & $\mathrm{~F}$ \\
\hline Asplanchna sieboldi (Leydig, 1854) & $* * *$ & $*$ & & $* *$ & * & $* * *$ & $* * *$ & $*$ & $* * *$ & $* * *$ & & * & 58 & $\mathrm{~F}$ \\
\hline Brachionus calyciflorus $\mathrm{P}$ & & & & & & & & & & $*$ & & & 3 & $\mathrm{R}$ \\
\hline Brachionus dolabratus Harri & $*$ & * & & & & & & & & $* * *$ & $* * *$ & $* * *$ & 31 & $\mathrm{c}$ \\
\hline Brachionus falcatus Zacharias, 1898 & & $* * *$ & $*$ & & $* *$ & & & & & & $* * *$ & $* *$ & 31 & $\mathrm{c}$ \\
\hline Brachionus mirus (Daday, 1905) & & & $*$ & & & & * & $*$ & $* *$ & $* *$ & $* * *$ & $* *$ & 33 & $\mathrm{c}$ \\
\hline Brachionus sp. & & & & $*$ & & & & & & & & & 3 & $\mathrm{R}$ \\
\hline Collotheca sp. & * & $* * *$ & $* *$ & $*$ & & & & & & & $* * *$ & * & 31 & $\mathrm{c}$ \\
\hline Conochius natans (Seligo, 1990) & $* * *$ & $* *$ & & * & & & * & & & & & & 19 & $\mathrm{R}$ \\
\hline Conochilus sp. & & & & * & $*$ & $* * *$ & & $*$ & & & & & 17 & $\mathrm{R}$ \\
\hline Conochilus coenobasis (Skorikov, 1914) & & $*$ & & * & & & & & & & $* *$ & $* *$ & 17 & $\mathrm{R}$ \\
\hline Conochilus unicor & $* * *$ & $* * *$ & $* * *$ & $* * *$ & $* * *$ & $* * *$ & $* * *$ & $* * *$ & $* * *$ & $* * *$ & $* *$ & $* * *$ & 97 & $\mathrm{Ct}$ \\
\hline Euchlanis dilatato & & $*$ & & & & $* * *$ & $* * *$ & $* *$ & $* * *$ & $* * *$ & $*$ & & 44 & $\mathrm{c}$ \\
\hline Filinia longi & $* *$ & $* *$ & & & & & & $*$ & * & $*$ & $* * *$ & $* *$ & 33 & $\mathrm{c}$ \\
\hline Filinia opoliensis ( $\mathrm{Za}$ & * & $* * *$ & $* *$ & $* *$ & & & $*$ & $* *$ & $*$ & $* * *$ & $* *$ & $* * *$ & 56 & $\mathrm{~F}$ \\
\hline Filinia sp. & & & & $*$ & & & & & & & & & 3 & $\mathrm{R}$ \\
\hline Gastropus sp. & * & $* * *$ & & $*$ & $* *$ & * & $* *$ & $*$ & & & & & 31 & $\mathrm{c}$ \\
\hline Hexartra intermedia (Weiszniewski, 1929) & $* * *$ & $* *$ & $* *$ & $* *$ & & & & & $*$ & $* * *$ & $* * *$ & $* * *$ & 56 & $\mathrm{~F}$ \\
\hline Kellicotia bostoniensis (Rousselete, 1908) & & & $*$ & $* * *$ & $* * *$ & & $* *$ & $* *$ & & $*$ & $* *$ & $*$ & 42 & $\mathrm{c}$ \\
\hline Keratella americana (Carlin, 1943) & $*$ & $* * *$ & $* * *$ & $* * *$ & $* * *$ & $* * *$ & $* * *$ & $* * *$ & $* * *$ & $* * *$ & $* * *$ & $* * *$ & 94 & $\mathrm{Ct}$ \\
\hline Keratella cochlearis (Gosse, 1851) & * & $* *$ & & $*$ & $* *$ & & $* *$ & $* *$ & $* *$ & $* * *$ & $* * *$ & $* * *$ & 58 & $\mathrm{~F}$ \\
\hline Keratella lenzi (Hauer, 1953) & $* *$ & $* * *$ & $* *$ & $* * *$ & $* * *$ & $* * *$ & $* * *$ & $* *$ & $*$ & * & & & 64 & $\mathrm{~F}$ \\
\hline Keratella sp. & & & & & & & $*$ & & $*$ & & & & 6 & $\mathrm{R}$ \\
\hline Keratella tecta Turn & & & & & & & & & $* *$ & $* * *$ & $* *$ & $* *$ & 25 & $\mathrm{c}$ \\
\hline Kerat & & & & & * & & & & & $*$ & & & 6 & $\mathrm{R}$ \\
\hline Kerat & & & & & & & & & $*$ & $* * *$ & $*$ & $*$ & 17 & $\mathrm{R}$ \\
\hline Lecane bulla (Gosse, & & & & & & & & & & & & $*$ & 44 & $\mathrm{c}$ \\
\hline Lecane luna (Müller, 1776) & & $* *$ & & & & & & & & & & & 6 & $\mathrm{R}$ \\
\hline Lecane leontina (Turner, 1892) & & & $*$ & & & & & & & & & & 3 & $\mathrm{R}$ \\
\hline Lecane lunaris (Ehrenberg 1832 & & & & & $*$ & $*$ & & & & & & * & 8 & $\mathrm{R}$ \\
\hline Lecane papuana Murr & & & & & & * & & $*$ & & & & & 6 & $\mathrm{R}$ \\
\hline Lecane proiecta Hauer, 1956 & $* * *$ & $* * *$ & $* * *$ & $* * *$ & $* *$ & * & & & $*$ & & & $*$ & 47 & $\mathrm{c}$ \\
\hline Lepadella $\mathrm{sp}$. & & & & & $*$ & * & $*$ & & & & & & 8 & $\mathrm{R}$ \\
\hline Plationus macracanthu: & & & & $*$ & $*$ & & & & & & & & 6 & $\mathrm{R}$ \\
\hline Polyarthra vulgar & & * & $* *$ & $* * *$ & $* *$ & & $* *$ & $* *$ & $* *$ & $*$ & $* *$ & $* *$ & 53 & $\mathrm{~F}$ \\
\hline Ptygura libe & & $*$ & & & & & & & & & & $* * *$ & 11 & $\mathrm{R}$ \\
\hline Synchaeta jollyii Shiel \& Koste, 1993 & $* * *$ & $* *$ & $* * *$ & $* *$ & $* * *$ & $* * *$ & $* *$ & $* *$ & $* * *$ & $* *$ & & & 69 & $\mathrm{~F}$ \\
\hline Testudinella patina (Hermann, 1783) & & $*$ & & & & & & * & & & & & 6 & $\mathrm{R}$ \\
\hline Trichocerca cylindrica (Sudzuki, 1956) & & $* * *$ & $*$ & $*$ & $* * *$ & $* *$ & & $*$ & $* * *$ & $* * *$ & $* * *$ & $* *$ & 61 & $\mathrm{~F}$ \\
\hline Trichocerca chatonni (de Beauchamp, 1907) & * & $* *$ & $* *$ & $* *$ & $*$ & $* *$ & $* *$ & $*$ & $* * *$ & $* *$ & $* * *$ & $*$ & 61 & $\mathrm{~F}$ \\
\hline Trichocerca similis grandis (Wierzejski, 1983) & * & $* *$ & $*$ & $* * *$ & $*$ & $* * *$ & $* * *$ & $* * *$ & $* *$ & & $* * *$ & $*$ & 64 & $\mathrm{~F}$ \\
\hline
\end{tabular}

Macrothrix cf. elegans. According to FeRNANDO (2002), the distinction between limnetic and littoral zooplankton is often not observed in the tropics, which explains the occurrence of these phytophylous organisms in the samples.

In the review by ESKINAZI-SANT'ANNA et al. (2005), 300 species of Rotifera were recorded in Minas Gerais, which only six of these species are registered for the Furnas reservoir and recorded in this study, what indicates that an update for rotifers in Minas Gerais is necessary. SEGERS \& Dumont (1995) identified 102 species of rotifers in 20 points distributed in the Broa reservoir, including sampling in the limnetic and littoral regions of the reservoir, while in this study 42 species were recorded, which only covered the limnetic region.

Jackknife 1 and Bootstrap estimators curves showed richness above that verified by the observed richness curve (Sobs) throughout the study. This result is common when non-parametric estimators are used to estimate the richness of zooplankton species (Dumont \& SEgERs, 1996; MuirHEAD et al., 2006; SousA, 2014). The estimator richness obtained 
by Jackknife 1 curve in BA1 station evidenced an asymptote, while for Bootstrap estimator curve, the asymptote was observed for BA1 and BA2 stations. The adjustments to other estimators curves showed a tendency to the formation of a asymptote, while for the uniques curves the asymptote was observed at all stations since $9^{\text {th }}$ sampling.

The estimator curves showed that only between $3 \%$ and $16 \%$ of environmental potential richness was not recorded and the sampling may be considered satisfactory. The richness species determined by the unique curves showed the asymptote beginning at $9^{\text {th }}$ successive monthly sampling. It indicates that the monthly sampling can be reduced to eight so that most of environment richness will be achieved in, in time scale. In the tropics, there is less variation in the seasonal succession of zooplankton species and species co-occur in both the summer and winter seasons (DUMONT, 1994). Thus, a smaller amount of samples is necessary and the time and sampling costs could be reduced.

The results obtained with the volume collected showed that $400 \mathrm{~L}$ are sufficient to record $90 \%$ of the environment richness, regardless of the sampling type (vertical hauls with a plankton net or suction motor pump). Also, another recommendation is to decrease from twelve to eight the monthly collections throughout the year, to goal a zooplankton limnetic species inventory, as the maximum number of species of Cladocera and Rotifera will be obtained in the waters of the Furnas reservoir in eight months.

Acknowledgements. We thank Professor Raoul Henry for his valuable comments which helped to improve the manuscript. This study was supported by "Eletrobras Furnas - Programa de P\&D Aneel".

\section{REFERENCES}

Begon, M.; Townsend, C. R. \& Harper, J. L. 2007. Ecologia: de Indivíduos a Ecossistemas. 4ed. Porto Alegre, Artmed. 752p.

Brito, S. L.; Maia-Barbosa, P. M. \& Pinto-Coelho, R. M. 2011. Zooplankton as an indicators of trophic conditions in two large reservoirs in Brazil. Lakes and Reservoirs: Reserch \& Management 16(4):253-264

COLWELL, R. K. 2009. EstimateS: statistical estimation of species richness and shared species from samples. Version 8.2 User's Guide and application. Available at $<$ http://viceroy.ebb.uconn.edu/EstimateS $>$. Accessed on 6 July 2012.

Colwell, R. K.; MaO, C. X. \& Chang, J. 2004. Interpolatin, extrapolatin, and comparing incidence-based species accumulation curves. Ecology 85:2717-2727.

Corgosinho, P. H. \& Pinto-Coelho, R. M. 2006. Zooplankton biomass, abundance and allometric patterns along a eutrophic gradient at Furnas Reservoir (Minas Gerais, Brazil). Acta Limnologica Brasiliensia 18(2):213-224

DAJOZ, R. 1983. Ecologia geral. Vozes, Petrópolis. 472p

De Melo, R. \& Hebert, P. D. N. 1994. A taxonomic reevaluation of North American Bosminidae. Canadian Journal of Zoology 72:1808-1825.

Dumont, H. J. 1994. The distribution and ecology of the fresh- and brackishwater medusae of the world. Hydrobiologia 272:1-12.

Dumont, H. J. \& Segers, H. 1996. Estimating lacustrine zooplankton species richness and complementarity. Hydrobiologia 341:125-132.

Elmoor-Loureiro, L. M. A. 1997. Manual de identificação de Cladóceros límnicos do Brasil. Brasília, Universa. 156p.

Eskinazi-Sant'Anna, E. M.; Maia-Barbosa, P. M.; Brito, S. \& RietzLER, A. C. 2005. Zooplankton Biodiversity of Minas Gerais State: a Preliminary Synthesis of Present Knowledge. Acta Limnologica Brasilensia 17(2):199-218.
FerNANDO, C. H. 2002. Zooplankton and tropical freshwater fisheries In: Fernando, C. H. ed. Guide to Tropical Freshwater Zooplankton. Identification, Ecology and Impacts on Fisheries. Leiden, Backhuys Publishers, p. 255-280.

Gotelli, N. \& Colwell, R. K. 2001. Quantifying biodiversity: procedures and pitfalls in the measurement and comparison of species richness. Ecology Letters 4:379-391.

HeCK, K. L.; Van-Belle, G. \& Simberloff, D. 1975. Explicit Calculation of the Rarefaction Diversity Measurement and the Determination of Sufficient Sample Size. Ecology 56(6):1459-1461.

Jersabek, C. D.; Segers, H. \& Morris, P. J. 2003. An illustrated online catalog of the Rotifera in the Academy of Natural Sciences of Philadelphia (version 1.0: 2003-April-8). Available at $<$ http://rotifer. ansp.org/rotifer.php>. Accessed on 14 December 2012.

KING, J. R. \& PORTER, S. D. 2005. Evaluation of Sampling Methods and Species Richness Estimators for Ants in Upland Ecosystems in Florida. Environmental Entomology 34(6): 1566-1578.

Koste, W. 1978. Rotatoria. Die RaÈdertiere Mitteleuropas, 2 vols. Berlim, Gebriider Bormtrager. 673p.

Kotov, A. A. 2009. A revision of Leydigia Kurz, 1875 (Anomopoda, Cladocera, Branchiopoda), and subgeneric differentiation within the genus. Zootaxa 2082:1-84.

Kozlowsky-SuZuKi, B. \& Bozelli, R. L. 1998. Avaliação da eficiência de três amostradores na estimativa de abundância de organismos zooplanctônicos na lagoa de Cabiúnas. In: Esteves, F. A. ed. Ecologia de lagoas costeiras do parque nacional da Restinga de Jurubatiba e do município de Macaé (RJ). Rio de Janeiro, Núcleo de Pesquisas Ecológicas de Macaé, UFRJ, p. 273-282.

Magurran, A. E. 2011. Medindo a diversidade biológica. Curitiba, Editora da UFPR. 261p.

Melo, A. S. 2008. O que ganhamos 'confundindo' riqueza de espécies e equabilidade em um índice de diversidade? Biota Neotropica 8(3):2127.

Muirhead, J. R.; Ejsmont-Karabin, J. \& Macisaac, H. J. 2006. Quantifying rotifer species richness in temperate lakes. Freshwater Biology 51:1696-1709.

OrLova-BienKowsKaJA, M. J. 1998. A revision of the cladoceran genus Simocephalus (Crustacea, Daphnidae). Bulletin of the Natural History Museum (Zoology series) 64(1):1-62.

Pinto-Coelho, R. M. 2004. Métodos de Coleta, Preservação, Contagem e Determinação de Biomassa em Zooplâncton de Águas Epicontinentais. In: Bicudo, C. E. M. \& Bicudo, D. orgs. Amostragem em Limnologia. São Carlos, RiMa, p.149-166.

Rocha, O.; SANtos-WisniewsKi, M. J. \& Matsumura-Tundisi, T. 2010 Check List de Cladocera de Água Doce do Estado de São Paulo. Biota Neotropica 11(1):571-591.

SANTOS, A. J. 2006. Estimativas de riqueza de espécies. In: CulLEN JR., L.; Valladares-Padua, C. \& Rudran, R. orgs. Métodos de Estudos em Biologia da Conservação e Manejo da Vida Silvestre. 2ed. Curitiba, Ed. Universidade Federal do Paraná. 652p.

Santos-Wisniewski, M. J.; Matsumura-Tundisi, T.; Negreiros, N. F.; Silva, L. C.; Santos, R. M. \& Rocha, O. 2011. O estado atual do conhecimento da diversidade dos Cladocera (Crustacea, Branchiopoda) nas águas doces do estado de Minas Gerais. Biota Neotropica 11(3):287-301.

SARMa, S. S. S.; NANDinI, S. \& Gulati, R. D. 2005. Life history strategies of cladocerans: comparisons of tropical and temperate taxa. Hydrobiologia 542:315-333.

Segers, H. 2007. Annotated checklist of the rotifers (Phylum Rotifera), with notes on nomenclature, taxonomy and distribution. Zootaxa 1564:1-104.

Segers, H. \& Dumont, H. J. 1995. 102+ rotifer species (Rotifera: Monogononta) in Broa reservoir (SP, Brazil) on 26 August 1994, with the description of three new species Hydrobiologia 316:183-197.

Sinev, A. Y. \& Elmoor-Loureiro, L. M. A. 2010. Three new species of chydorid cladocerans of subfamily Aloninae (Branchipoda: Anomopoda: Chydoridae) from Brazil. Zootaxa 2390:1-25.

Smirnov, N. N. 1998. A revision of the genus Camptocercus (Anomopoda, Chydoridae, Aloninae). Hydrobiologia 386:63-83.

Sousa, F. D. R.; Elmoor-Loureiro, L. M. A.; Mendonça-Galvão, L. \& Pujol-Luz, J. R. 2014. Evaluation of a new sampling method for assessing Cladocera richness (Crustacea, Branchiopoda) in macrophyterich wetlands. Annales de Limnologie 50:143-153. 